\title{
Computer "Experiments" on Classical Fluids. I. Thermodynamical Properties of Lennard-Jones Molecules*
}

\author{
LOUP VERLET \\ Belfer Graduate School of Science, Yeshiva University, New York, New York
}

(Received 30 January 1967)

\begin{abstract}
The equation of motion of a system of 864 particles interacting through a Lennard-Jones potential has been integrated for various values of the temperature and density, relative, generally, to a fluid state. The equilibrium properties have been calculated and are shown to agree very well with the corresponding properties of argon. It is concluded that, to a good approximation, the equilibrium state of argon can be described through a two-body potential.
\end{abstract}

\section{INTRODUCTION}

$\mathrm{T}$ HE "exact" machine computations relative to classical fluids have several aims: It is possible to realize "experiments" in which the intermolecular forces are known; approximative theories can thus be unambiguously tested and some guidelines are provided to build such theories whenever they do not exist. The comparison of the results of such computations with real experiments is the best way to obtain insight into the interaction between molecules in the high-density states.

The Monte Carlo method initiated by the Los Alamos group $^{1}$ is a first example of these "exact" methods. It amounts to a direct computation of the integrals involved in the canonical averages. It is easy to carry out, with the inconvenience, however, of providing no information on the time properties of the system.

The dynamics of an isolated system can also be considered and used to calculate time averages and time-dependent properties. The case of hard spheres and hard spheres surrounded by a square well has been extensively studied by Alder et $a l .{ }^{2}$ In the case of a twobody interaction simulating more closely the interaction between the molecules, it is possible to integrate directly the equation of the motions of about a thousand particles, as brilliantly demonstrated by Rahman. ${ }^{3}$ The present paper presents some of the results which have been obtained, using a technique inspired by Rahman's work, for a system of 864 particles interacting through a Lennard-Jones potential.

In Sec. II we give some technical details on the method which we use; in particular, we describe a bookkeeping device that cuts the computing time by a factor of the order of 10.

In Sec. III we give and discuss the results obtained for the pressure, the internal energy, the high-frequency elastic moduli, and the isotopic separation factor. These

\footnotetext{
* Supported by the U. S. Air Force Office of Scientific Research Grant No. 508-66.

†Permanent address: Faculté des Sciences, Laboratoire de Physique Théorique et Hautes Energies, Bâtiment 211, 91-Orsay.

1 W. W. Wood and F. R. Parker, J. Chem. Phys. 27, 720 (1957).

2 B. J. Alder and T. E. Wainwright, J. Chem. Phys. 33, 1439 (1960).

${ }^{3}$ A. Rahman, Phys. Rev. 136, A405 (1964).
}

results, summarized in Table I, are sufficiently numerous to allow a comparison on the whole density range of the fluid state and on a wide temperature range which essentially excludes the extremely high temperatures. The over-all agreement is surprisingly good. It appears that the many-body forces, if they are at all important, behave so as to realize an effective interaction which is state independent to a good approximation.

The correlation functions are described and discussed in a separate paper. ${ }^{4}$ The formalism necessary to express the fluctuations in the microcanonical ensemble was discussed recently. ${ }^{5}$ It can be applied to calculate the derivatives of the thermodynamic functions (e.g., the specific heat and $\partial p / \partial \rho$ ) in terms of fluctuations averaged over time. The results are not very precise and will only be presented as an illustration of these theoretical considerations. ${ }^{6}$

The results on the time-dependent properties will be reported later.

\section{DESCRIPTION OF THE COMPUTER EXPERIMENTS}

We consider a system of 864 particles, enclosed in a cube of side $L$, with periodic boundary conditions interacting through a two-body potential of the Lennard-Jones type

$$
V(r)=4\left((\sigma / r)^{12}-(\sigma / r)^{6}\right) .
$$

This potential is cut at $r_{v}=2.5 \sigma$ in most of our experiments, or, in some of them at $r_{v}=3.3 \sigma$. The problem is to integrate the equation of motion

$$
\frac{d^{2} \mathbf{r}_{i}}{d t^{2}}=\sum_{j \neq i} \mathbf{f}\left(r_{i j}\right) .
$$

We choose the following units: The lengths are expressed in units of $\sigma(\sigma=3.405 \AA$ for argon), and the energies in units of $\epsilon\left(\epsilon=119.8^{\circ} \mathrm{K}\right.$ for argon $) .^{7}$ The thermodynamic quantities will thus be measured in the

${ }^{4} \mathrm{~L}$. Verlet (to be published).

5 J. L. Lebowitz and J. K. Percus, Phys. Rev. 124, 1673 (1961).

6 J. L. Lebowitz, J. K. Percus, and L. Verlet, Phys. Rev. 153, 250 (1967).

${ }^{7}$ A. Michels and H. Wijker, Physica 15, 627 (1949). 
usual "reduced" units. The time unit is chosen so that $m=48 \epsilon \sigma^{-2}$; it turns out to be, for argon, equal to $3 \times 10^{-13} \mathrm{sec}$. This time is of the order of the kinetic relaxation times of the system in the case considered in this paper. With this in mind, we have, for the force acting on particle $i$ in the $x$ direction,

$$
f_{x}\left(r_{i j}\right)=m\left(x_{i}-x_{j}\right)\left(r_{i j}^{-14}-0.5 r_{i j}^{-8}\right) .
$$

To integrate (2), we use the very simple algorithm

$$
\mathbf{r}_{i}(t+h)=-\mathbf{r}_{i}(t-h)+2 \mathbf{r}_{i}(t)+\sum_{j \neq i} \mathbf{f}\left(r_{i j}(t)\right) h^{2},
$$

where $h$ is the time increment which we take equal to 0.032 . This is practically the value chosen by Rahman (i.e., $10^{-14} \mathrm{sec}$ in the case of argon). We have checked that this time increment is adequate and even superfluously small in most cases. For instance, for $T=1.38$, $\rho=0.55$ (i.e., temperature just above critical, density almost twice critical), we have performed two integrations up to the time $t=4$. In one case we have taken $h=0.032$, in the other $h=0.016$, with the same initial conditions. The difference in position at time $t$ is typically of the order of 0.001 and the difference in the thermodynamic quantities, at the same final time, amounts to $1 / 10000$. In this kind of calculation, most of the time is spent in computing the force. If no special devices are introduced, we must, at each step, compute $\frac{1}{2} N(N-1)$ terms, most of which turn out to be zero. We introduce the following bookkeeping device ${ }^{8}$ which cuts the computing time by a factor of the order of 10 : Every $n$th step, we compute all the $\frac{1}{2} N(N-1)$ distances, and, given a particle $i$, we make a table of all the particles which are within a distance $\boldsymbol{r}_{M}$ of that particle. Then, for the next $n-1$ steps in time, we take into account only the particles in the tables. There is no error as long as $r_{M}$ is sufficiently larger than $\boldsymbol{r}_{v}$ so that no particle outside the table traverses the "skin" of depth $r_{M}-r_{v}$ and gets into the range $r_{v}$ of the potential. The feasibility of such a procedure can be easily appreciated by giving some orders of magnitude: Let $\bar{v}$ be the root-mean-square velocity in our units, it is typically of the order of 0.3 ; if this is so, no error is made as long as

$$
r_{M}-r_{v} \lesssim n \bar{v} h
$$

If, for instance $n=16, n \bar{v} h=0.15$. By choosing $r_{M}=3.3$ for $r_{v}=2.5$, the condition (5) is largely met, and at the same time the "skin" depth stays reasonably small. We have checked, by following some systems for several hundred steps in time, that no difference at all was observed when $n$ was reduced. Moreover, the conservation of the total energy and of the total velocity, which stays of the order of $10^{-7}$, is a guarantee of the soundness of the whole procedure.

${ }^{8}$ Some time-saving tricks have been considered before: See Ref. 2 and, for the hard-sphere case, A. Rotenberg [New York University Report No. NYO-1480-3, 1964 (unpublished)].
With this device, the time spent for an integration step at the density 0.45 is about $12 \mathrm{sec}$ on the UNIVAC 1107 of the Faculté des Sciences, Orsay, where the first calculations were made, and ten times less (with careful machine coding of the time-consuming subroutines) on the CDC 6600 of New York University, where the greatest part of the results reported here were obtained.

With that machine, a typical "experiment" takes about $1 \mathrm{~h}$. It goes as follows: The positions are initially taken, in general, at the nodes of a face-centered-cubic lattice which has the desired density, and the velocities are chosen at random with a Gaussian probability law. Three hundred steps in time are sufficient, in general, to reach equilibrium. The computation is then carried on for 1200 steps in time (this corresponds, for argon, to $\left.1.2 \times 10^{-11} \mathrm{sec}\right)$. The main part of the computation concerns the study of equilibrium quantities (thermodynamic functions: temperature, pressure, internal energy, specific heat, etc.; time-independent correlation functions) and of nonequilibrium quantities (velocity autocorrelation function, elastic constants, viscosities, heat conductivity, etc.). The necessary technical details will be given when these results are reported.

\section{THERMODYNAMIC QUANTITIES}

\section{A. Temperature}

At each step in time the velocities are calculated simply by the formula

$$
\mathbf{v}_{i}(t)=\left[\mathbf{r}_{i}(t+h)-\mathbf{r}_{i}(t-h)\right] / 2 h .
$$

The temperature is, at time $t, \frac{2}{3}$ of the kinetic energy, in our units

$$
T=16 \sum_{i} v_{i}^{2} / N
$$

The error entailed by the use of (6) is of the order of $1 / 1000$. This error is of no consequence, except that it gives rise to small irregularities in the total energy which should be otherwise strictly constant. The temperature, averaged over the time, is affected by a statistical error of the order of 0.004 .

\section{B. Pressure}

The pressure is calculated from the virial theorem

$$
\frac{p}{\rho k T}=1-\frac{1}{6 N k T}\left\langle\sum_{i} \sum_{j>i} \frac{\partial v_{i j}}{r_{i j}} \frac{\rho}{\partial r_{i j}}\right\rangle-\frac{\rho}{6 k T} \int_{r_{v}}^{\infty} \underset{r-g}{\partial r} g(\mathbf{r}) d \mathbf{r} .
$$

The second term of (8) is the time average of the virial. The last term is a correction term which takes into account the effect on the pressure of the tail of the potential which has been neglected in the dynamics.

The influence on the main term of (8) of the tail of the potential, which has been neglected in the dynamics can be appreciated by considering that cutoff tail as a 
TABLE I. List of thermodynamical results: $\rho$ is the particle density, and $T$ the temperature $; \beta p / \rho$ is the compressibility factor, $\Omega_{0}{ }^{2}$ is equal to $\left\langle\nabla^{2} V\right\rangle / m, K_{\infty}$ and $G_{\infty}$ are the infinite-frequency bulk and shear moduli, respectively, $K_{\infty} / G_{\infty}$ their ratio, and $r_{v}$ the distance at which the potential is cut. Reduced units are used throughout.

\begin{tabular}{|c|c|c|c|c|c|c|c|c|}
\hline$\rho$ & $T$ & $\beta p / \rho$ & $U^{i}$ & $\Omega_{0}^{2}$ & $K_{\infty}$ & $G_{\infty}$ & $K_{\infty} / G_{\infty}$ & $r_{v}$ \\
\hline 0.88 & 1.095 & 3.48 & -5.66 & 23.3 & 58.2 & 32.1 & 1.81 & 2.5 \\
\hline 0.88 & 0.94 & 2.72 & -5.84 & 21.4 & 52.4 & 29.8 & 1.76 & 2.5 \\
\hline 0.88 & 0.591 & -0.18 & -6.53 & 17.3 & 42.5 & 26.2 & 1.62 & 2.5 \\
\hline 0.85 & 2.889 & 4.36 & -4.25 & 35.1 & 90.7 & 44.5 & 2.04 & 2.5 \\
\hline 0.85 & 2.202 & 4.20 & -4.76 & 30.9 & 77.4 & 39.3 & 1.97 & 2.5 \\
\hline 0.85 & 1.214 & 3.06 & -5.60 & 22.4 & 54.7 & 30.3 & 1.81 & 2.5 \\
\hline 0.85 & 1.128 & 2.78 & -5.69 & 21.5 & 52.2 & 29.3 & 1.78 & 2.5 \\
\hline 0.85 & 0.880 & 1.64 & -5.94 & 18.8 & 45.0 & 26.4 & 1.70 & 2.5 \\
\hline 0.85 & 0.782 & 0.98 & -6.04 & 17.8 & 42.1 & 25.3 & 1.66 & 2.5 \\
\hline 0.85 & 0.786 & 0.99 & -6.05 & 17.8 & 42.2 & 25.4 & 1.67 & 2.5 \\
\hline 0.85 & 0.760 & 0.78 & -6.07 & 17.5 & 41.4 & 25.0 & 1.66 & 2.5 \\
\hline 0.85 & 0.719 & 0.36 & -6.12 & 17.0 & 39.9 & 24.4 & 1.63 & 2.5 \\
\hline 0.85 & 0.658 & -0.20 & -6.19 & 16.4 & 38.3 & 23.8 & 1.61 & 2.5 \\
\hline 0.85 & 0.591 & -1.20 & -6.26 & 15.5 & 35.7 & 22.7 & 1.57 & 2.5 \\
\hline 0.75 & 2.849 & 3.10 & -4.07 & 26.8 & 59.3 & 30.2 & 1.96 & 2.5 \\
\hline 0.75 & 1.304 & 1.61 & -5.02 & $\cdots$ & 36.1 & 20.9 & 1.73 & 2.5 \\
\hline 0.75 & 1.069 & 0.90 & -5.19 & 15.4 & 32.0 & 19.3 & 1.65 & 2.5 \\
\hline 0.75 & 1.071 & 0.89 & -5.17 & 15.4 & 31.6 & 19.1 & 1.66 & 3.3 \\
\hline 0.75 & 0.881 & -0.12 & -5.31 & 14.1 & 27.8 & 17.5 & 1.56 & 2.5 \\
\hline 0.75 & 0.827 & -0.54 & -5.38 & 13.2 & 26.9 & 17.2 & 1.53 & 2.5 \\
\hline 0.65 & 2.557 & 2.14 & -3.78 & & 35.8 & 19.3 & 1.85 & 3.3 \\
\hline 0.65 & 1.585 & 1.25 & -4.23 & $\ldots$ & 25.5 & 15.0 & 1.70 & 2.5 \\
\hline 0.65 & 1.036 & -0.11 & -4.52 & 11.5 & 19.7 & 12.7 & 1.55 & 3.3 \\
\hline 0.65 & 0.900 & -0.74 & -4.61 & 10.4 & 17.8 & 11.9 & 1.50 & 2.5 \\
\hline 0.55 & 2.645 & 1.63 & -3.24 & $\ldots$ & 23.0 & 12.7 & 1.80 & 2.5 \\
\hline 0.5426 & 3.26 & 1.86 & -3.00 & $\ldots$ & 26.1 & 13.9 & 1.88 & 2.5 \\
\hline 0.5426 & 1.404 & 0.57 & -3.63 & $\ldots$ & 14.2 & 9.0 & 1.58 & 2.5 \\
\hline 0.5426 & 1.326 & 0.42 & -3.66 & $\ldots$ & 13.9 & 8.9 & 1.56 & 3.5 \\
\hline 0.5 & 1.36 & 3.40 & -3.38 & 8.5 & 11.4 & 7.4 & 1.54 & 2.3 \\
\hline 0.45 & 4.625 & 1.68 & -2.22 & 14.9 & 21.4 & 11.2 & 1.91 & 2.5 \\
\hline 0.45 & 2.935 & 1.38 & -2.60 & 11.0 & 14.8 & 8.3 & 1.79 & 2.5 \\
\hline 0.45 & 1.744 & 0.74 & -2.90 & 8.20 & 11.0 & 6.7 & 1.64 & 2.5 \\
\hline 0.45 & 1.764 & 0.76 & -2.89 & 8.20 & 11.0 & 6.7 & 1.64 & 2.5 \\
\hline 0.45 & 1.710 & 0.74 & -2.95 & 8.20 & 11.0 & 6.7 & 1.64 & 2.5 \\
\hline 0.45 & 1.552 & 0.75 & -2.98 & 7.7 & 10.1 & 6.4 & 1.58 & 2.5 \\
\hline 0.4 & 1.462 & 0.41 & -2.72 & $\cdots$ & 7.27 & 4.77 & 1.53 & 2.5 \\
\hline 0.4 & 1.424 & 0.38 & -2.73 & $\ldots$ & 7.22 & 4.75 & 1.52 & 3.3 \\
\hline 0.35 & 1.620 & 0.58 & -2.31 & 5.8 & 5.70 & 3.72 & 1.54 & 2.5 \\
\hline 0.35 & 1.418 & 0.40 & -2.21 & $\cdots$ & 4.92 & 3.31 & 1.49 & 2.5 \\
\hline
\end{tabular}

weak long-range perturbation. ${ }^{9}$ The effect on the main term of (8) is quite small: It amounts to 0.006 in the one case studied $(\rho=0.75, T=1.05)$. It is smaller than the statistical error which is generally of the order of 0.01 when 1200 steps in time are considered. The main error comes certainly from the correction term which is very large at high density and low temperature where it is of the order of 1 for $r_{v}=2.5$.

The replacement of $g(r)$ by 1 in the correction term leads, for $r_{v}=2.5$, to an error which may reach 0.05 at the highest density and lowest temperature considered here. Such a replacement is, however, not necessary as the extrapolated values of $g(r)^{4}$ may be used.

As a whole, we believe that the over-all error on $p / \rho k T$ is probably of the order of 0.01 around the critical density and may reach 0.05 in the high-density and low-temperature region.

The results so far obtained have been gathered in Table I. Fig. 1 shows $p / \rho k T$ for some isochores at high

\footnotetext{
${ }^{9} \mathrm{~J}$. Lebowitz (private communication).
}

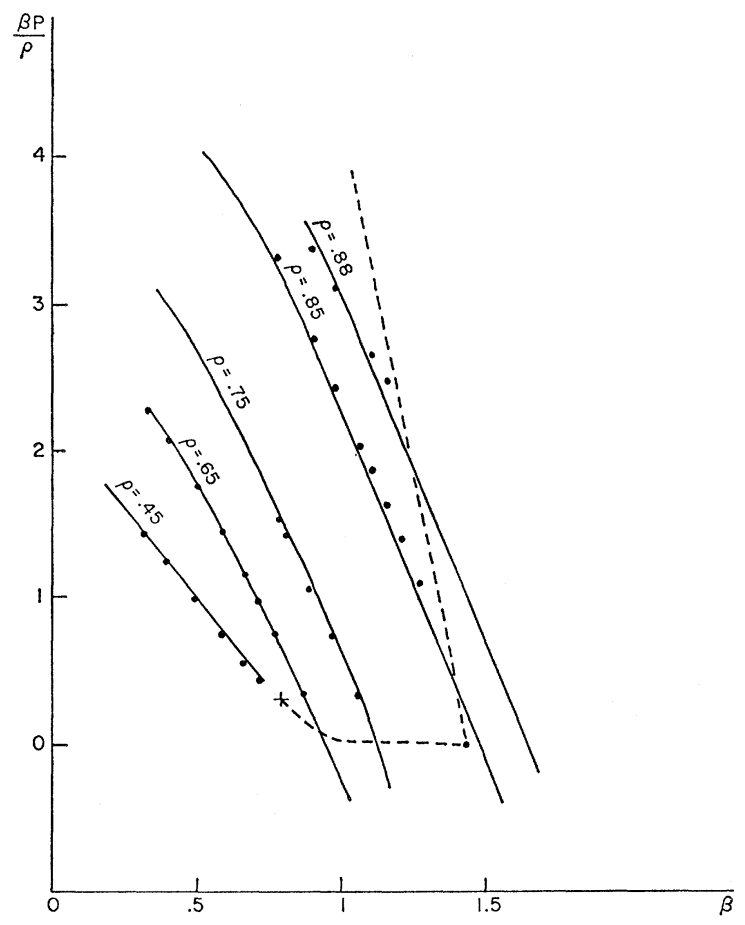

FIG. 1. The compressibility factor $\beta p / \rho$ as a function of $\beta$ for the isochores $\rho=0.88,0.85,0.75,0.65$, and 0.45 (solid lines), as compared with the experimental data for argon (Refs. 10-12) (dots). The dashed curves correspond to the gas-liquid and solidfluid coexistence lines.

density ( $\rho=0.88,0.85,0.75,0.65$, and 0.45$)$, and the comparison of the equation of state determined by various experimental groups in Amsterdam, ${ }^{7,10}$ Toronto, ${ }^{11}$ and Louvain. ${ }^{12}$

The over-all agreement between "theory" and experiment is surprisingly good: It appears that the LennardJones potential is a quite satisfactory interaction as far as the equilibrium properties of argon are concerned. It is to be noted that if, instead of argon, xenon were chosen for the comparison, the agreement would not be so good. For instance, with, for $T=1.35, \rho=0.75$, the value of $\beta p / \rho$ from molecular dynamics is 0.86 ; the same quantity is equal to 0.86 in argon. For xenon (with the reduction parameters $\left.\epsilon / k=225.3^{\circ} \mathrm{K}, \sigma=4.07 \AA\right)$, the value 1.05 is obtained. ${ }^{10}$

On Fig. 2 are represented three isotherms: One at high temperature $(T=2.74)$ where comparison can be made with the results obtained a long time ago by Wood and Parker, ${ }^{2}$ and also with a Lennard-Jones potential through the Monte Carlo technique; the lowtemperature isotherm $T=1$; and the isotherm $T=1.35$, which is also an isotherm for which Monte Carlo com-

10 J. M. H. Levelt, Physica 26, 361 (1960).

11 W. Van Witzenburg, University of Toronto dissertation (unpublished); copy available in Dissertation Abstracts 25, 1268 (1964).

${ }^{12}$ A. Van Itterbeck, O. Verbeke, and K. Staes, Physica 29, 742 (1963). 


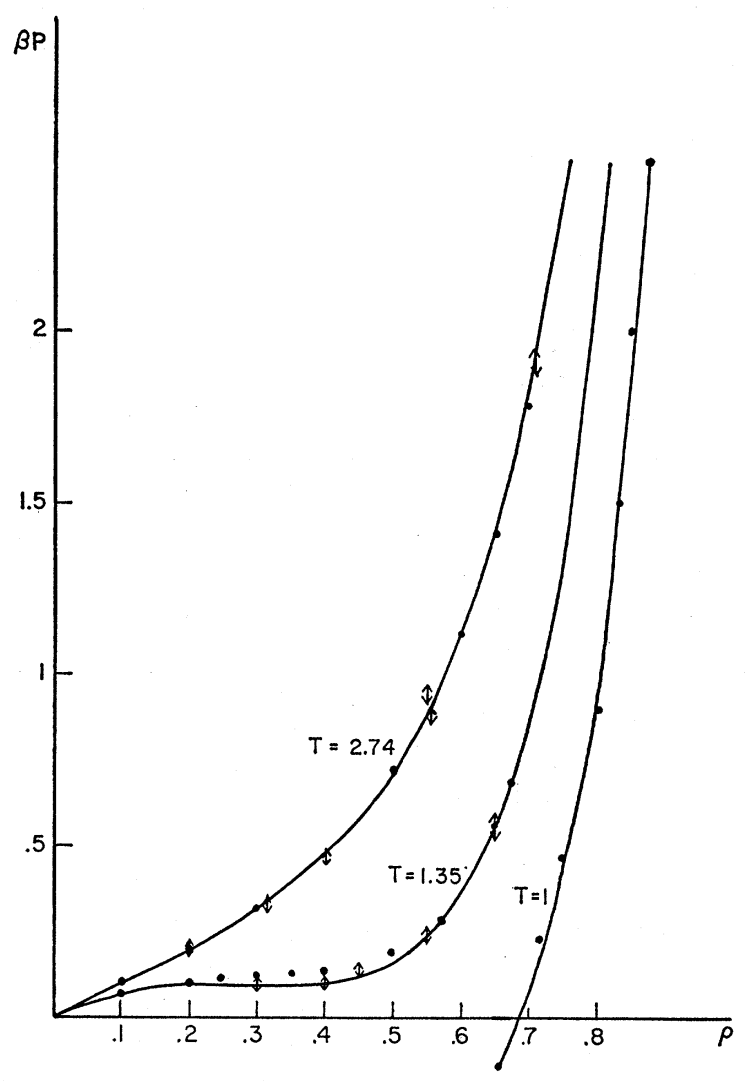

FIG. 2. For the isotherms $T=2.74, T=1.35$, and $T=1, \beta p$ is represented as a function of $\rho$. The low-density parts of the curves were obtained with the help of the PY and PY II equations (Ref. 13). The arrows show the results of Monte Carlo calculations (Refs. 1, 13). The dots are the experimental results in argon (Refs. 10-12).

putations have been made. ${ }^{13}$ That isotherm will be used below to examine the question of the critical constants.

For low values of the pressure, our computation may describe metastable states: The periodic boundary prevents the occurrence of inhomogeneities of a size larger than the side of the box, so that the formation of a two-phase system is very much hindered. The low pressure part of the isochores at $\rho=0.85$ and 0.88 has been obtained by "cooling" a liquid configuration: That is, we start from a spatial distribution relative to the liquid state, and a Gaussian initial velocity distribution. Equilibrium configurations corresponding to supercooled liquids may thus be reached. We have also started from solid-like spatial configurations, with again Gaussian velocity distributions. The temperature $T_{0}$ corresponding to this initial velocity is progressively raised until "melting" occurs. Our criterion ${ }^{14}$ for melting is based on the evolution in time of $\rho_{k}=\sum_{i} \cos k x_{i}$ : There $k$ corresponds to the smallest vector of the reciprocal lattice, i.e., $k=4 \pi / a$, where $a$ is the side of

${ }^{13} \mathrm{D}$. Levesque and L. Verlet, Physica (to be published).

${ }_{14} \mathrm{P}$. Nozières (private communication). unit cell of the original fcc lattice, which, in the $x$ direction extends from 0 to $L$. For a solid, $\rho_{k}$ is of the order of $N$. It oscillates around 0 with an amplitude of the order of $\sqrt{ } N$ for a liquid. If we admit that the metastable states of the solid are short-lived (as compared to the total time of the computation, i.e. $1.2 \times 10^{-11} \mathrm{sec}$ for argon), we can find by trial and error the melting temperature. For $\rho=0.85$ a solid configuration is still observed for $T=0.695$. It corresponds to a value of the initial temperature $T_{0}=1.308$. For $T_{0}=1.366$, melting does occur. Experimentally (see Fig. 1) melting occurs at $T=0.704$. For $\rho=0.88$, we have a solid for $T=0.825$; this corresponds to $T_{0}=1.545$. With $T_{0}=1.67$, melting is reached. Experimentally, melting takes place for $T=0.82$ at that density. The agreement with experiment is seen to be good.

\section{Critical Constants}

Because of computational errors, the precise determination of the critical constants is difficult. We believe, however, that a careful examination of the data warrants the following discussion and conclusions.

(a) The extrapolation of the isochores towards the critical temperature yields results which are in good agreement with the Monte Carlo results of Levesque and Verlet ${ }^{13}$ (108 particles, about 300000 configurations). Those results, for $T=1.35$, are shown in Fig. 2 and in Table II.

(b) The PY II equation leads at $\rho=0.45$ and $\rho=0.5$ to values of $\beta p / \rho$ which are definitely too low. The trend to give too low values for the quantities at intermediate density is already noticeable at $\rho=0.4$, as may be seen from Table III, although one is at the border of computational errors. If the values yielded by the PY II equation are corrected by 0.005 at $\rho=0.35$, and by 0.02 at $\rho=0.4$-and this seems to be the most that can be expected-the following critical constants are obtained: $T_{c}=1.32, \rho_{c}=0.32$, and $\beta_{c} p_{c} / \rho_{c}=0.3$, instead of those obtained from the PY II equation, $T_{c}=1.36, \rho_{c}=0.36$, and $\beta_{c} p_{c} / \rho_{c}=0.36$. We expect the critical constants to be situated somewhere between those limits.

TABLE II. Values of $\beta p / \rho$ for the isotherm $T=1.35$ obtained from the PY and PY II equations (Ref. 13), Monte Carlo computations (M.C.) (Ref. 13), extrapolation of the molecular dynamics (M.D.) results, 5-term virial series (Ref. 15), its Padé approximant, and experiment on argon (Ref. 10).

\begin{tabular}{lccccccc}
\hline \hline$\rho$ & PY & PY II & M.C. & M.D. & 5 Vir. & Padé & Exp. \\
\hline 0.2 & 0.510 & 0.50 & & & 0.506 & 0.505 & 0.527 \\
0.3 & 0.396 & 0.36 & $0.36 \pm 0.03$ & & 0.377 & 0.374 & 0.404 \\
0.35 & 0.376 & 0.30 & & $0.32 \pm 0.02$ & 0.350 & 0.343 & 0.368 \\
0.4 & 0.386 & 0.27 & $0.28 \pm 0.03$ & $0.29 \pm 0.02$ & 0.351 & 0.338 & 0.349 \\
0.45 & 0.434 & 0.25 & $0.33 \pm 0.03$ & $0.30 \pm 0.02$ & 0.382 & 0.359 & 0.353 \\
0.5 & 0.532 & 0.30 & & $0.33 \pm 0.03$ & 0.446 & 0.406 & 0.388 \\
0.55 & 0.692 & 0.45 & $0.44 \pm 0.03$ & $0.42 \pm 0.02$ & 0.545 & 0.481 & 0.471 \\
0.65 & 1.256 & 1.22 & $0.86 \pm 0.05$ & $0.82 \pm 0.03$ & 0.860 & 0.709 & 0.863 \\
\hline \hline
\end{tabular}


TABLE III. Values of $\beta p / \rho$, of the inverse compressibility (Ref. 4), and of the interaction part of the integral as given by the molecular dynamics (M.D.), the PY II, and the PY equation for $T=1.46, \rho=0.4$.

\begin{tabular}{ccrr}
\hline & M.D. & PY II & \multicolumn{1}{c}{ PY } \\
\hline$\beta p / \rho$ & $0.41 \pm 0.01$ & 0.40 & 0.51 \\
$\beta \partial p / \partial \rho$ & 0.54 & 0.53 & 0.49 \\
$U^{i}$ & $-2.72 \pm 0.01$ & -2.72 & -2.71 \\
\hline \hline
\end{tabular}

(c) The five virial series ${ }^{15}$ gives values of $\beta p / \rho$ which are clearly too high by 0.015 at $\rho=0.3$, by 0.05 at $\rho=0.35$, and by 0.06 at $\rho=0.4$. The difference then diminishes and changes sign at high density. The Padé approximant shows the same behavior but a little less accentuated. We conclude that one or several virial coefficients after the fifth one must be negative, and that some higher ones must be positive again.

The critical point deduced by Barker et al..$^{15}$ from their series is situated at $T_{c}=1.29, \rho_{c}=0.26$, and $\beta_{c} p_{c} / \rho_{c}=0.35$. The critical density is quite low, a consequence of the too low pressures at intermediate densities.

(d) Table IV summarizes the situation: There a comparison with experiment on argon is made, which shows that there exists a discrepancy of at least $5 \%$ on the critical temperature. This raises the following problem: It is known nowadays ${ }^{16,17}$ that the low-density data are not well fitted by a Lennard-Jones potential; a potential with a deeper bowl and a weaker tail, such as the recently determined Kihara potential, ${ }^{17}$ seems much more appropriate. It would seem natural to guess that using such a potential will improve the situation in the critical region. The reverse is true: Levesque and Vieillard-Baron, ${ }^{18}$ using the PY II equation, find a critical temperature of 1.42 (with the same units as before). The blame for this discrepancy may be put on many-body forces. Even at density around the critical region, a relatively large amount of those forces would be needed. It appears that these many-body forces

TABLE IV. Critical constants obtained from the PY II equation (Ref. 13), from the PY II equation corrected as explained in Sec. III.A, from the 5-term virial series (Ref. 15) and from experiment (Ref. 10).

\begin{tabular}{clll}
\hline \hline & \multicolumn{1}{c}{$T_{c}$} & \multicolumn{1}{c}{$\rho_{c}$} & \multicolumn{1}{c}{$\beta_{c} p_{c} / \rho_{c}$} \\
\hline PY II & $1.36 \pm 0.04$ & $0.31 \pm 0.03$ & $0.36 \pm 0.03$ \\
PY II & & & \\
Maximum correction & 1.32 & 0.31 & 0.30 \\
5-term virial & 1.29 & 0.26 & 0.352 \\
Experiment & 1.26 & 0.297 & 0.316 \\
\hline
\end{tabular}

${ }^{15}$ J. A. Barker, P. J. Leonard, and A. Pompe, J. Chem. Phys. 44, 4266 (1966).

${ }^{16}$ E. A. Guggenheim and M. C. McGlasham, Proc. Roy. Soc. (London) 225A, $456(1960)$; R. J. Munn and J. Alder, J. Chem. Phys. 43, 3998 (1965) ; J. Rowlinson, Discussions Faraday Soc. 40, 19 (1965).

17 J. A. Barker, W. Fock, and F. Smith, Phys. Fluids 7, 429 (1964).

${ }^{18} \mathrm{D}$. Levesque and J. Vieillard-Baron (to be published). should then manifest themselves in the form of an interaction which is clearly state dependent at high density; and this seems to be contradicted by the excellence of the fit obtained in the present paper.

\section{Internal Energies}

The part of the internal energy due to the interaction $U^{i}$ is calculated as the time average of the sum of the interparticle interaction energies. A tail correction is included, as for the pressure and the energy; the over-all error appears to be of the order of 0.02 . The agreement between our results and the (not too extensive) experimental data ${ }^{10}$ is satisfactory, although the calculated internal energies are a little larger in magnitude than the experimental one. For instance, for $\rho=0.45$, at $T=2.935, U_{\text {calc }}{ }^{i}=-2.60, U_{\exp }{ }^{i}=-2.49$; at the same density, for $T=1.764, U_{\text {calc }}{ }^{i}=-2.89, U_{\exp }{ }^{i}=-2.84$. At higher densities, the same trend is noticeable, for $\rho=0.5426, T=1.404, U_{\text {calc }^{i}}=-3.63, U_{\exp }{ }^{i}=-3.55$. It appears, roughly speaking, that the Lennard-Jones potential is slightly too deep.

\section{E. High-Frequency Elastic Moduli}

Zwanzig and Mountain ${ }^{19}$ have shown that in simple fluids the infinite-frequency bulk modulus $K_{\infty}$ and the infinite-frequency shear modulus $G_{\infty}$ can be expressed in terms of the radial distribution function; and that, furthermore, in the case of the Lennard-Jones potential, the necessary integrals can be expressed in terms of the pressure and of the internal energy, so that $G_{\infty}$ and $K_{\infty}$ are simply given by the following expressions:

$$
\begin{aligned}
& G_{\infty}=3 p-2 \rho T-(24 / 5) \rho U^{i}, \\
& K_{\infty}=(5 / 3) G_{\infty}+2(p-\rho T) .
\end{aligned}
$$

Results for $K_{\infty}, G_{\infty}$, and the ratio $K_{\infty} / G_{\infty}$ are given in Table I. When the temperature is low, the internalenergy effects predominate and $K_{\infty} / G_{\infty}$ is near $(5 / 3)$, the value obtained from Cauchy condition for solids.

We may compare the "exact results" given in Table I with those obtained by Zwanzig and Mountain who used (9) and (10), and the experimental data of Levelt, ${ }^{10}$ supposing that the interaction between argon atoms is exactly the Lennard-Jones potential. The good agreement between those results and ours shows againalthough this proof is not independent of the preceding ones - that the Lennard-Jones potential is appropriate to describe argon in the temperature and density domain considered in the present paper.

\section{F. Isotopic Separation Factor}

The separation faction in a liquid-gas mixture of isotopes of argon can be expressed ${ }^{20}$ in terms of the

${ }^{19}$ R. Zwanzig and R. D. Mountain, J. Chem. Phys. 43, 4464 (1965).

${ }^{20}$ G. Casanova, A. Levi, and N. Terzi, Physica 30, 937 (1964). 


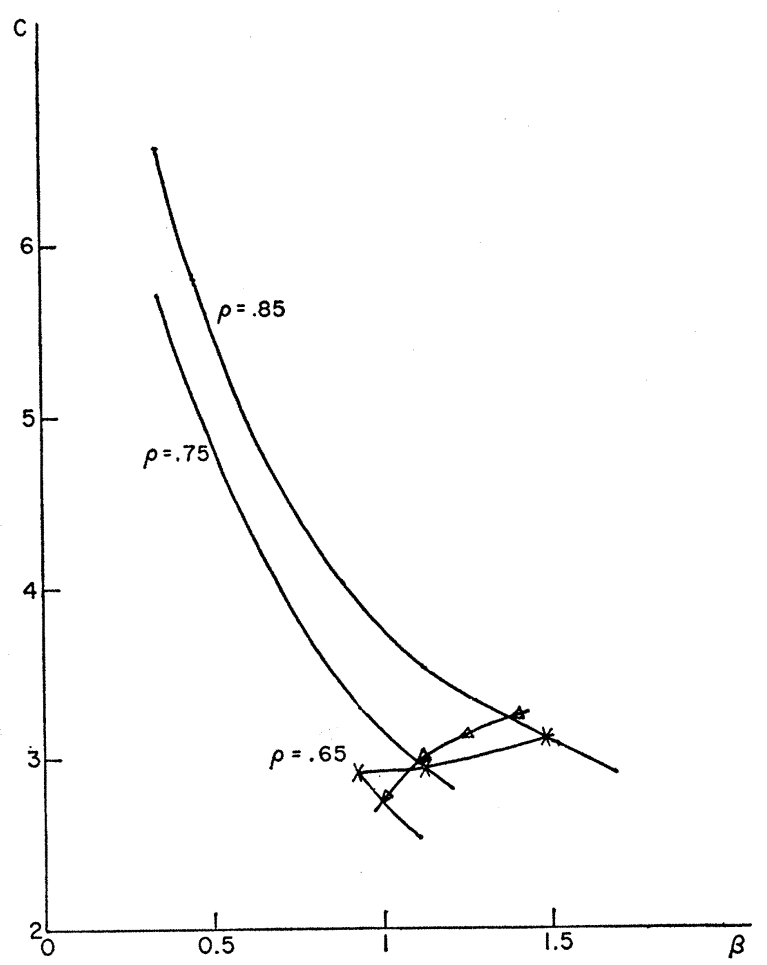

FIg. 3. The solid lines show the quantity $C=\Omega_{0}{ }^{2} / 2 \pi \rho m$ $=\left\langle\Delta^{2} V\right\rangle / 96 \pi \rho$ for the isochores $\rho=0.85, \rho=0.75$, and $\rho=0.65$, as a function of $\beta$. The crosses represent the intercepts of those isochores with the experimental liquid-gas coexistence line. It should be compared with the isotopic separation factor in argon (Ref. 20) (triangles).

average value of the Laplacian of the potential $\left\langle\nabla^{2} V(r)\right\rangle$. The quantity $\Omega_{0}^{2}=(1 / M)\left\langle\nabla^{2} V(r)\right\rangle$, which is also important in the time development of the system, has been calculated, and the values we obtained are shown in Table I. The quantity $C=\Omega_{0}^{2} / 2 \pi \rho$ is plotted for several isochores in Fig. 3. We have used experimental information (pressure-versus-density curve for the coexistence curve, as shown on Fig. 1), to locate the temperature of the coexistence line on the isochores. It may be seen easily, however, that this introduces very little arbitrariness. A good fit of the experiment is obtained, as seen from Fig. 3.

\section{CONCLUSIONS}

We have shown, using Rahman's work as a starting point, how it is possible to integrate the equations of motion of about a thousand particles in a relatively easy way. The first application of this tool is the thermodynamic study of a fluid composed of atoms interacting through a Lennard-Jones potential. The striking result of this study is the over-all agreement between the results thus obtained and the thermodynamics of real argon. It is likely that those results can still be slightly improved and that a two-body potential can fit the experimental data with a large degree of success.

\section{ACKNOWLEDGMENTS}

The author is very grateful for the hospitality of Yeshiva University where most of this work was done. It is a particular pleasure to thank Professor Joel L. Lebowitz; numerous long discussions have been both inspiring and stimulating in the series of studies whose results we have begun to describe. We are grateful also to D. Levesque, J. Vieillard-Baron, and D. Schiff for help and discussions. Last, but not least, we must acknowledge the profuse computing opportunities and the warm welcome in the Courant Institute of New York University, where the largest part of the computations were performed, and where the author benefitted from interesting and helpful discussions with Professor J. Percus and Dr. M. Kalos. 